\title{
Hard-Rod Behavior in Dense Mesophases of Semiflexible and Rigid Charged Viruses
}

\author{
Eric Grelet \\ Université de Bordeaux, CNRS, Centre de Recherche Paul-Pascal, \\ 115 Avenue Schweitzer, 33600 Pessac, France \\ (Received 28 February 2014; published 23 June 2014)
}

\begin{abstract}
We report on the phase behavior of a model system of colloidal rodlike particles, namely, the filamentous $f d$ viruses, in the dense liquid crystalline states. After determining the phase boundaries as a function of the added salt, we propose a renormalization of the phase diagram accounting for the screened electrostatic repulsions between the particles through an effective hard-rod diameter. Including explicitly counterion condensation, we show that our heuristic model captures the main feature of the nematic-to-smectic phase transition of long hard rods, i.e., its universal packing fraction. The importance of rod flexibility on the relative stability of the different concentrated mesophases is also demonstrated, evidencing, in particular, the existence of a smectic-B phase in between the smectic-A and the columnar phases.
\end{abstract}

DOI: 10.1103/PhysRevX.4.021053

\section{INTRODUCTION}

Suspensions of colloidal particles self-organize spontaneously into different states, displaying phase transitions that can be driven by entropy alone. Such mesoscopic particles usually exhibit phase behavior very similar to atoms or molecules and, therefore, are often considered as paradigms in condensed matter physics [1-3]. The features of model system of colloids stem from both the simplicity of their interaction, which is mainly steric, and from their ability to be observed at the single-particle level by optical microscopy $[4,5]$. In this respect, colloidal particles with nonspherical shapes (such as rodlike and disklike particles) have attracted considerable attention because of their outstanding faculty to form liquid crystals [6-11]. The nematic state exhibits long-ranged orientational order, while the smectic and columnar phases additionally possess positional order in one and two dimensions, respectively [12]. In this study, we explore the phase behavior in the smectic and columnar phases of highly monodisperse charged rods, the semiflexible filamentous bacteriophages, $f d$ [5]. We determine the experimental phase diagram in the regime of high virus concentrations as a function of the added salt ionic strength $I_{s}$, and we propose a renormalization of the phase diagram accounting for the rod electrostatic repulsion, where counterions stemming from the viral particles have to be taken into account via a virus effective charge. Our results are compared with theoretical predictions and computer simulations, showing that rodlike viruses behave nearly as hard rods, even in the highly ordered dense states.

* grelet@crpp-bordeaux.cnrs.fr

Published by the American Physical Society under the terms of the Creative Commons Attribution 3.0 License. Further distribution of this work must maintain attribution to the author(s) and the published article's title, journal citation, and DOI.
Subject Areas: Soft Matter, Statistical Physics

Such a description of the electrostatic interactions between viral rods could be particulary relevant for the recently reported virus-based colloidal membranes [13-15], which are polymorphic unilamellar self-assemblies reminiscent of the bulk smectic organization. In the present work, we evidence two smectic phases, a smectic-A phase and a newly reported smectic-B phase, and we also investigate the effect of rod flexibility on the mesophase stability thanks to a stiffer viral mutant. Furthermore, we show that the location of the smectic-to-columnar ( $\mathrm{Sm}-\mathrm{Col})$ transition is independent of the bending flexibility, in contrast with the nematic-to-smectic $(\mathrm{N}-\mathrm{Sm})$ transition that is strongly affected by the rod rigidity.

\section{THEORETICAL CONSIDERATIONS}

Rodlike particles are usually modeled as hard impenetrable cylinders of contour length $L$, diameter $D$, and volume $v_{0}=\pi L D^{2} / 4$. Driven by a gain in excluded volume, i.e., by a gain of entropy, they self-organize into different mesophases by increasing rod density. The first theory of ordered assemblies of rods with purely hard-core repulsive interactions was proposed by Onsager to describe the isotropic liquid-to-nematic (I-N) transition [16]. It represents a paradigm of a very general class of phase transitions that are entropy driven [17]. For such systems, the steric repulsion between pairs of particles evaluated by the second virial coefficient $B_{2}$ is directly related to the excluded volume $\left\langle V_{\text {excl }}\right\rangle[12,16]$, where the brackets account for the average over the particle's orientational distribution. For two cylinders, the general expression of their excluded volume is given by

$$
V_{\mathrm{excl}}=2 L^{2} D|\sin \gamma|+2 \pi D^{2} L
$$

where $\gamma$ is the angle between their two long axes. For a uniform fluid of $N$ particles in a volume $V$ at temperature $T$, 
the particle-particle interaction is expressed through the excess contribution to the Helmoltz free energy and is given at the second virial level by [18]

$$
F_{\mathrm{ex}} / N k_{B} T \simeq \rho B_{2}=\frac{1}{2} \frac{\left\langle V_{\mathrm{excl}}\right\rangle}{v_{0}} \phi,
$$

where the volume fraction $\phi$ is related to the rod number density $\rho=N / V$ via $\phi=\rho v_{0}$. In the long-rod limit $(L \gg D)$, the excluded volume scales as $V_{\text {excl }} \propto L^{2} D$ near the I-N transition, which yields $F_{\text {ex }} \propto \frac{L}{D} \phi$. The phase diagram is therefore expected to depend only on the parameter $\frac{L}{D} \phi$, as rigorously predicted by Onsager for the I-N transition [16]:

$$
\phi_{\mathrm{I}-\mathrm{N}} \simeq 4 \frac{D}{L}
$$

In the case of parallel hard rods, and neglecting the rodend effect, the excluded volume reduces to $V_{\text {excl }}^{\|} \propto L D^{2} \propto v_{0}$ according to Eq. (1). $F_{\mathrm{ex}}^{\|}$therefore becomes independent of the geometrical properties of the particles and is only a function of the rod-packing fraction $\phi$. In this long-rod limit and with the approximation of perfectly aligned particles, the N-Sm transition volume fraction is then expected to be universal, i.e., occurring at a fixed volume fraction, $\phi_{\|}=c s t$, no matter what the particle size is [19-21]. For the N-Sm transition, the low-density approximation becomes poor, and higher-order virial coefficients have to be added a priori to the free energy. This is usually done following the Parsons-Lee scheme, which is a twobody resummation extending the semiempirical CarnahanStarling approximation for hard spheres [22,23]. The second virial coefficient $B_{2}$ is then weighted by a density prefactor that only depends on the volume fraction $\phi$ : Such rescaling of the free energy quantitatively improves the predictions but does not qualitatively affect the result shown above, i.e., the universal packing fraction of the $\mathrm{N}-\mathrm{Sm}$ transition for slender rods. In the literature, the reduced density $\phi_{\mathrm{N}-\mathrm{Sm}}^{*}=\phi_{\mathrm{N}-\mathrm{Sm}} / \phi_{\mathrm{cp}}$, where $\phi_{\mathrm{cp}}=\pi / 2 \sqrt{3}$ is the close-packed volume fraction of parallel cylinders, is found within a range of values between 0.36 and 0.47 by means of various approximate theories, including parallel or freely rotating rods, as well as simulations [19-21,24-29].

\section{EXPERIMENTS}

Our experimental system of rods is the filamentous $f d$ virus, which has been widely used as a colloidal model system in soft condensed matter [5,30-32]. The bacteriophage $f d$, which is an almost-1-micron-length ( $L=$ $0.88 \mu \mathrm{m})$ semiflexible charged rodlike particle with a diameter of $D=7 \mathrm{~nm}$ and a persistence length of $P=$ $2.8 \mu \mathrm{m}$, has a molecular weight of $M_{w}=1.64 \times 10^{7} \mathrm{~g} / \mathrm{mol}$ and is formed by single-stranded DNA around which about 2700 coat proteins are helicoidally wrapped $[33,34] . f d Y 21 M$ is a viral mutant that differs from wildtype $f d$ only by a single-point mutation of the 21 st amino acid of each major coat protein, changing from tyrosine in $f d$ to methionine in $f d Y 21 M$ [35]. This results in a stiffer phage, with a persistence length of $P=9.9 \mu \mathrm{m}$ [34], a contour length of $L=0.92 \mu \mathrm{m}$ [36], and a similar charge density [37]. The virus concentration $C_{\text {virus }}$ was measured using spectrophotometry with absorption coefficients of 3.84 and $3.63 \mathrm{~cm}^{2} / \mathrm{mg}$ at $269 \mathrm{~nm}$ for $f d$ and $f d Y 21 M$, respectively [34], and it is related to the volume fraction $\phi$ by $\phi=\rho v_{0}=C_{\text {virus }} N_{A} / M_{w} \times \pi L D^{2} / 4$, where $N_{A}$ is Avogadro's number. The $f d$ charge density is 3.4 e/protein at $\mathrm{pH} 8.1$ [38], giving a number of negative charges per virus of $Z_{\text {virus }}=9180$ [39]. The ionic strength $I_{s}$ and the $\mathrm{pH}$ associated with the added salt are monitored thanks to a TRIS-HCl-NaCl buffer against which dilute virus suspensions were extensively dialyzed. It is worth mentioning that the $\mathrm{CO}_{2}$ that dissolves from the air in the buffer cannot be neglected, and it significantly increases the ionic strength, of about $0.9 \mathrm{mM}$ for an analytical concentration of the TRIS buffer of $10 \mathrm{mM}$ at $\mathrm{pH} 8.1$ [40]. After dialysis, the virus suspensions were ultracentrifuged at about $200000 \mathrm{~g}$ for 3 hours. The viruses were resuspended in the same buffer, and different dilutions were placed in glass capillary tubes of diameter $1.5 \mathrm{~mm}$. The smectic phase was mainly identified by its optical iridescence stemming from the diffraction by visible light of the around-1-micron-thick smectic layers [36]. The chiral nematic phase was determined by its typical fingerprint texture observed by a polarizing microcopy [37], and the signature of the columnar mesophase was provided by the hexagonal positional order probed by small-angle x-ray scattering (SAXS) [31]. SAXS experiments were performed with a NanoStar-Bruker AXS setup, working at a wavelength of $1.54 \AA(\mathrm{Cu} \mathrm{K} \alpha$ emission) and with a sample-to-detector distance of $1.06 \mathrm{~m}$. The instrumental resolution is given by the full width at half maximum (FWHM) of the direct beam and is about $0.05 \mathrm{~nm}^{-1}$. For the study at $I_{s}=20 \mathrm{mM}, f d$ and $f d Y 21 M$ suspensions were prepared in flat capillaries in order to perform both smectic-layer-spacing measurements by differential-interference contrast microscopy and SAXS investigations on the same samples.

\section{RESULTS AND DISCUSSIONS}

Figure 1 depicts the phase diagram of $f d$ viruses in the dense regime where the boundaries between the chiral nematic, the smectic, and the columnar mesophases are indicated [41]. As expected, the location of the phase transitions occurs at higher virus concentrations, $C_{\text {virus}}$, when increasing the ionic strength because of the screening of the electrostatic repulsions [42]. 


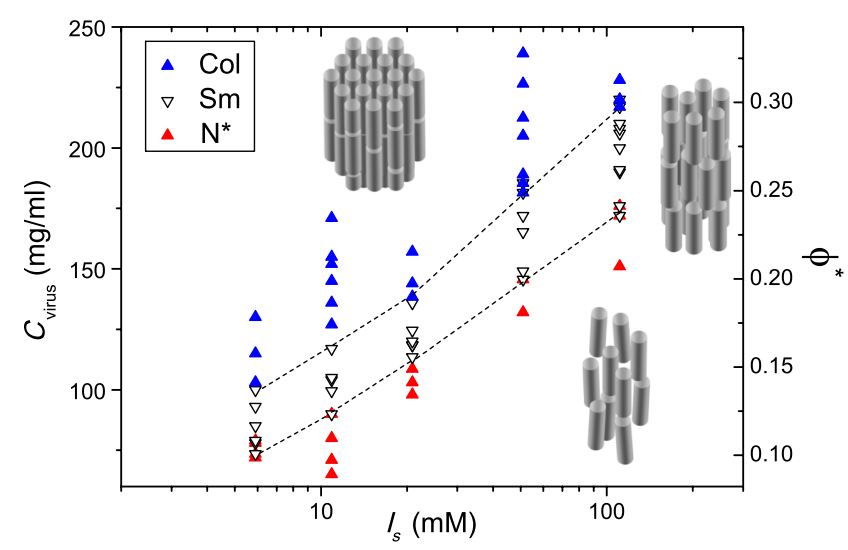

FIG. 1. Experimental phase diagram of the dense liquidcrystalline states of $f d$ virus suspensions. The reduced rod packing fraction $\phi^{*}$ is plotted as a function of the added salt $I_{s}$. The dashed lines are a guide for the eye to indicate the N-Sm and $\mathrm{Sm}-\mathrm{Col}$ phase transitions, where the smectic range (open white symbols) includes both the smectic-A and the smectic-B mesophases (see text).

\section{A. Ion condensation and virus effective charge}

The extent of the electric double layer surrounding each charged particle is characterized by using the Debye screening length $\kappa^{-1}$ [43]:

$$
\kappa^{-1}=\left(8 \pi \ell_{b} N_{A} I\right)^{-1 / 2},
$$

where $\ell_{b}$ is the Bjerrum length (of about $0.71 \mathrm{~nm}$ in water at room temperature), $N_{A}$ is Avogadro's number, and $I$ is the ionic strength, defined by

$$
I=\frac{1}{2} \sum_{i} n_{i} Z_{i}^{2} .
$$

In Eq. (5), the sum is taken over all types of mobile (or free) microions of molar concentration $n_{i}=\rho_{i} / N_{A}$ and of charge number $Z_{i}$, i.e., added salt ions and counterions compensating the virus surface charge [43-46]. In concentrated samples, the contribution of counterions originating from the charged colloids cannot be neglected, and this leads to an effective (or renormalized) charge of the viruses, which is also called charge condensation [47]. In its simplest form, condensation theory ignores the details of the ion distribution near the polyelectrolyte and assumes that a fraction of the rod charge will be neutralized because of the nonspecific binding with counterions. Let us call $Q$ the fraction of free counterions. In units of molarity, the ionic strength $I$ can then be expressed for monovalent microions (1:1 salt) by

$$
I \equiv I_{\mathrm{tot}}=I_{c}+I_{s}=\frac{1}{2} Q n_{c}+n_{s},
$$

with $n_{c}=C_{\text {virus }} \times Z_{\text {virus }} / M_{w}$ and $n_{s}$ the molar concentration of added salt. In the limit of infinite dilution and for a charged rod with vanishingly small radius $\left(\kappa_{0} R \ll 1\right.$, with $\kappa_{0}$ the Debye screening length associated with the added salt $I_{s}$ ), the uncondensed counterion fraction is given by $Q_{0}=\frac{1}{\lambda_{\text {bare }} \ell_{b}}=0.14$ according to the Manning criterion [48], where $\lambda_{\text {bare }}=Z_{\text {virus }} / L$ is the linear charge density of $f d$ virus. Such an approximation is valid neither for concentrated colloidal suspensions nor in the range of ionic strength studied here $\left(I_{s}=6\right.$ to $\left.110 \mathrm{mM}\right)$ for which the cylinder radius is comparable to the width of the diffuse ion atmosphere surrounding the particle, i.e., $\kappa_{0} R \sim 1$. More generally, addressing the question of the effective charge of dense assemblies of highly charged rods remains tricky, and it depends a priori on the rod diameter, the rod volume fraction, and the amount of added salt. In this context, Manning has recently developed an analytical approach for different geometries of particles, where the fraction of condensed ions results from the balance between enthalpic electrostatic binding and their dissociation entropy [49]. In the specific case of charged cylinders in the presence of added salt such as $\kappa_{0} R \sim 1$, Manning derived the following expression for the effective line charge density, $\lambda_{\text {eff }}$ :

$$
\lambda_{\text {eff }} \ell_{b}=-\ln \left(\frac{\kappa_{0}}{\lambda_{\text {bare }}}\right) \kappa_{0} R \frac{K_{1}\left(\kappa_{0} R\right)}{K_{0}\left(\kappa_{0} R\right)},
$$

where $K_{0}(x)$ and $K_{1}(x)$ are modified Bessel functions of the second kind. The fraction of free ions can thus be written as

$$
Q=\frac{\lambda_{\text {eff }}}{\lambda_{\text {bare }}}
$$

with $0 \leq Q \leq 1$ and is plotted in Fig. 2. It confirms the major role of counterions in the electrostatic properties and shows that ion condensation diminishes with increasing salt, ultimately being completely unbound at sufficiently high salt. The main restriction of the Manning model applies to colloids in the infinite dilution limit. In order to

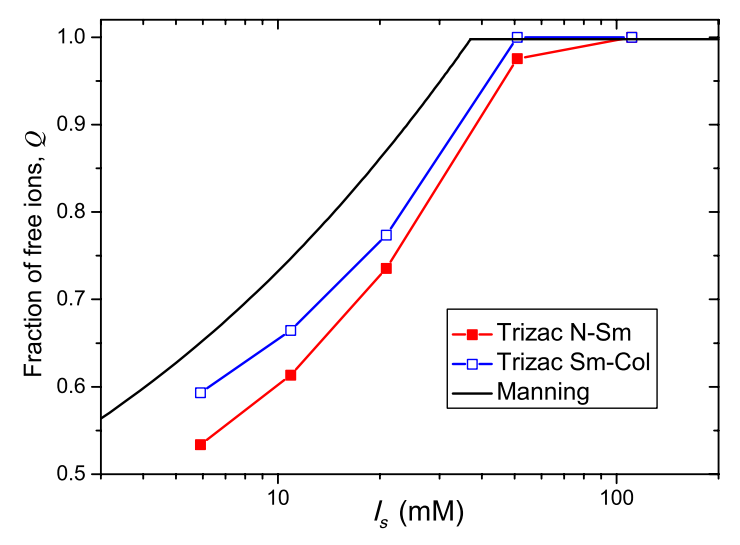

FIG. 2. Fraction of free ions $Q$ as a function of the added salt $I_{s}$ obtained from calculations of Manning [49] (black line) and Trizac et al. [53] (open and full squares). 
account for the finite particle volume fraction, a mean field Poisson-Bolztmann theory within a cell model [50] has to be used. This approach replaces the many-body problem of charged colloidal suspensions by a simpler one-particle system and has been shown to successfully describe the electrostatic interactions with monovalent ions. In this framework, analytical expressions have been derived for charge renormalization of macroions by Trizac et al. [51-53]. Their approximation becomes exact in the colloidal limit $\kappa_{0} R \gg 1$, but it has been shown to be a good estimate down to $\kappa_{0} R \gtrsim 1$, corresponding to the specific case studied here. For the sake of clarity, the equations for determining the virus effective charge are not explicitly written here, but the details can be found in Ref. [53]. The result of the computation is shown in Fig. 2 for both volume fractions corresponding to the $\mathrm{N}-\mathrm{Sm}$ and $\mathrm{Sm}-\mathrm{Col}$ phase transitions. Compared with the Manning model, a very similar dependence of the fraction of free ions is found, where all the counterions are dissociated from the rod surface above a given amount of added salt. Despite their slight quantitative difference, the very good consistency between the two main available analytical models found in the literature makes us confident about capturing the underlying counterion contribution in the $f d$ virus suspensions.

\section{B. Effective diameter and renormalized phase diagram}

In order to effectively account for the electrostatic repulsion in our statistical model, which only contains the excluded-volume interaction [Eq. (2)], the bare diameter $D$ has to be rescaled by a thicker effective hard-rod diameter $D_{\text {eff }}$. Many attempts were made for rodlike particles $[11,30,42,44,45,54]$, mainly focusing on the isotropic liquid phase for which an effective diameter can be strictly defined at the level of the second virial approximation according to Stroobants et al. $[55,56]$. This approach allows for a semiquantitative agreement with charged filamentous viruses at the I-N transition [57,58], but it fails to account for the N-Sm transition because such an expression of $D_{\text {eff }}$ is intrinsically ill defined for parallel rods $[29,56]$. Therefore, and because a rigorous theory including an electrostatic interaction at a fundamental level is still missing for charged rods organized in highly ordered states, we have chosen a heuristic approach in the spirit of Onsager, who mentioned in his seminal paper that [16] "[...] the effective range of the electrostatic repulsion will be a modest multiple of the screening distance $\kappa^{-1}$," which means, for $D_{\text {eff }}$,

$$
D_{\text {eff }}=D+\alpha \kappa^{-1},
$$

with $\alpha$ a free parameter and $\kappa^{-1}$ the Debye screening length associated with the total ionic strength $I_{\text {tot }}$ [Eq. (6)]. The effective diameter, and consequently the effective volume fraction, is then a function of the added salt $I_{s}$ and the virus concentration $C_{\text {virus }}$, both known experimentally, as well as a function of the fraction of free ions $Q$ determined through a charge-condensation model, and of the free parameter $\alpha$. Since $f d$ viruses exhibit both a high aspect ratio $(L / D \gg 1)$ and a near-parallel configuration close to the N-Sm transition, as shown by an orientational order parameter $S \sim 1$ [59], the nematic-to-smectic transition volume fraction is expected to be universal [20], i.e., $\phi_{\text {eff }}^{*}=$ cst. By fitting, accordingly, the effective volume fraction as a function of the added salt $I_{s}$ using $\alpha$ as the only fit parameter, we obtain the renormalization of the N-Sm for both models of charge condensation (Manning [49] and Trizac et al. [53]): $\phi_{\mathrm{N}-\mathrm{Sm}}^{*}=0.46$ and 0.43 , respectively, as shown in Fig. 3. Both values, which are close to each other, are found within the experimental error bars, and they are, in general, in good agreement with the different theoretical predictions [19,20,24-27,29], as well as the most recent Monte Carlo simulations on long rigid rods, giving $\phi_{\mathrm{N}-\mathrm{Sm}}^{*} \in[0.46,0.47]$ [28]. When virus flexibility is considered, it is expected that the N-Sm transition shifts to higher rod densities when compared to perfect rigid hard rods $[28,36]$. Such an effect is indeed observed for the stiffer $f d Y 21 M$ mutant $(P / L \sim 10)$ for which the effective volume fraction $\phi_{\mathrm{N}-\mathrm{Sm}}^{*}$ decreases by about $9 \%$, when determined with the same set $(Q, \alpha)$ of parameters as those used for the wild-type semiflexible virus. More details on the effect of rod stiffness can be found in Sec. IV C.

In Fig. 4, we plot the effective diameter $D_{\text {eff }}$ obtained for the renormalized phase diagram (Fig. 3) at the N-Sm transition. By applying the same procedure including charge condensation and keeping unchanged the parameter $\alpha$, the effective diameter at the I-N transition is found to be in good agreement with the one obtained by Onsager theory

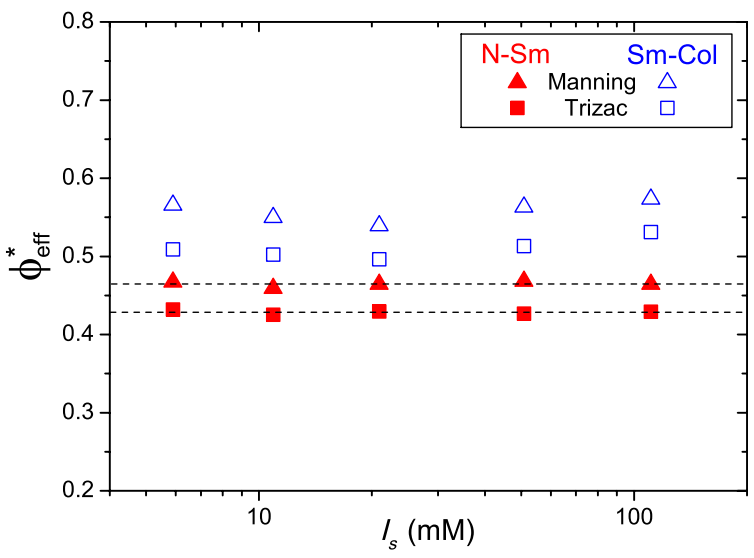

FIG. 3. Renormalized phase boundaries of N-Sm (full red symbols) and Sm-Col (open blue symbols) transitions for $f d$ virus suspensions. The reduced effective volume fraction $\phi_{\mathrm{eff}}^{*}$ is obtained with the effective rod diameter $D_{\text {eff }}(\alpha, Q)=D+\alpha \kappa^{-1}$ [Eq. (9)], where the variable fraction $Q$ of uncondensed virus counterions is given by the models of Manning [49] (triangles) and Trizac et al. [53] (squares), as shown in Fig. 2. 


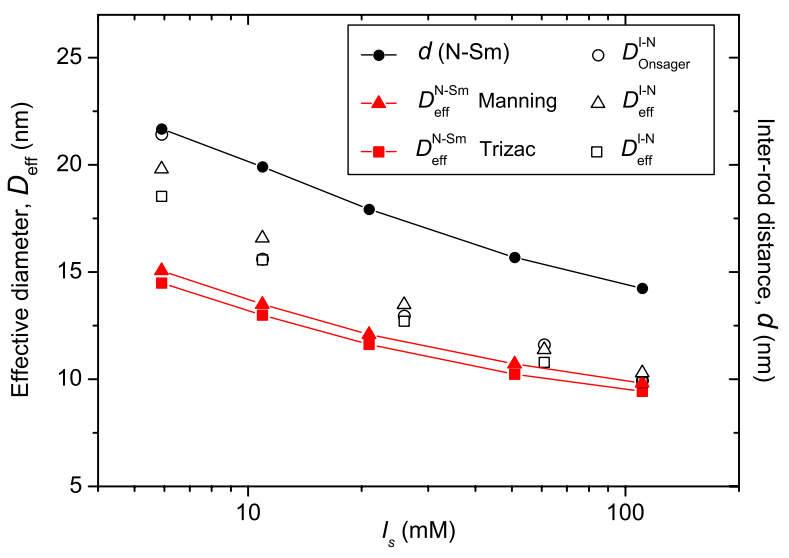

FIG. 4. Effective hard-rod diameter $D_{\text {eff }}$ [Eq. (9)] used for the renormalization of the phase diagram (Fig. 3) with the fraction of free ions $Q$ calculated according to the models of Manning [49] (triangles) and Trizac et al. [53] (squares). $D_{\text {eff }}$ at the N-Sm transition (red symbols) is shown to always be smaller than the corresponding inter-rod distance $d$ obtained by SAXS (black dots). $D_{\text {eff }}$ at the I-N transition (open symbols) obtained by the two charge condensation models described above and from Onsager theory for semiflexible rods $[57,60]$.

for semiflexible rods [57,60], validating the approach developed here. When our effective diameter is compared to the inter-rod $d$ distance obtained by SAXS measurements via $d=\frac{2}{\sqrt{3}} \frac{2 \pi}{q_{100}}$ [31], it results in $D_{\text {eff }}<d$ for all transitions, and whatever the added salt $I_{s}$-so a fortiori whatever the ionic strength $I-$, meaning that there is no overlap of the electric double layer between rods. This observation supports the hard-rod behavior of $f d$ viruses in the dense regime. Contrary to previous claims [5,30,42], we show that the rod charge can be quantitatively taken into account via an effective diameter $D_{\text {eff }}$, demonstrating, in particular, the importance of charge condensation (which is sometimes neglected because of its complexity and usually ignored for dilute suspensions, but which must be taken into account in concentrated regime) to properly account for the screened electrostatic interactions.

Even if there has not been a full theoretical demonstration to date, we tried to apply the same approach of renormalization to the Sm-Col transition. In this case and contrary to both the N-Sm and the I-N transitions, this procedure works poorly, as shown in Fig. 3. Experimentally, it becomes more and more difficult to get homogeneous samples as the ionic strength and the rod concentration increase, generating data dispersion. Beyond our heuristic approach, some effects such as dispersion forces have been neglected and could possibly play a role as the rod density increases. Van der Waals interactions are mainly independent of ionic strength and usually exhibit the largest attraction for parallel-rod configurations [56], therefore favoring the smectic phase stability [61]. The short range $(0-10 \mathrm{~nm})$ of this attraction may have some influence, especially on the Sm-Col transition, considering the short surface-to-surface distance between viruses at the highest ionic strength (Fig. 4). Some effective attraction due to many-body effects could also be invoked [62], decreasing the location of the phase transitions as well. Nevertheless, these effects remain limited and can be seen as a perturbation of the prevailing hard-rod behavior of $f d$ suspensions.

\section{Influence of rod flexibility}

As already mentioned above, rod stiffness represents a major parameter in the relative stability of the smectic phase with respect to the nematic and the columnar phases $[63,64]$. If the smectic phase is easily characterized experimentally by its optical iridescence stemming from its layered organization [Figs. 5(a) and 5(b)], the nature of the order within the layers has to be probed by SAXS. Figure 5(c) shows the scattered intensity corresponding to liquidlike order (dotted black line) featuring a SmA phase [41], and to hexagonal positional order (red line) by slightly increasing the rod packing. Combined with the layer ordering along the rods, this defines a $\mathrm{SmB}$ phase found for both the semiflexible $f d$ and the stiff $f d Y 21 M$ viruses, schematically represented in Fig. 5(d) [11,12]. In the SmB phase, the positional correlation length $\zeta_{\perp}$ normal to the rod-long axes is long ranged, being only limited by the instrumental resolution [Fig. 6(b)]. It distinguishes SmB from the hexatic columnar mesophase of filamentous viruses, which exhibits, furthermore, liquidlike order along the column axes [31]. The hexatic feature of the columnar mesophase is provided by the short-range hexagonal translational order, which is broken by the presence of dislocations. Such intrinsic defects stem from the

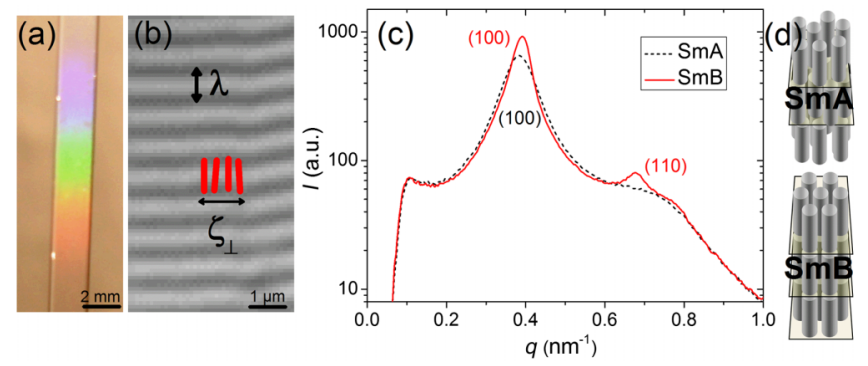

FIG. 5. (a) Iridescence of macroscopic smectic sample illuminated by a white light source. (b) Optical texture of the smectic phase observed by differential interference contrast microscopy evidencing the layer spacing $\lambda$. The red rods schematically represent the $f d$ viruses within the layers, and the associated positional correlation length $\zeta_{\perp}$ normal to their long axis. (c) Scattered intensity obtained by SAXS probing the translational order within the smectic layers showing the evolution from liquidlike (dotted black line) to hexagonal positional (solid red line) order by slightly increasing the rod concentration. This corresponds to the $\mathrm{SmA}$ and $\mathrm{SmB}$ phases, respectively, as schematically represented in (d). 


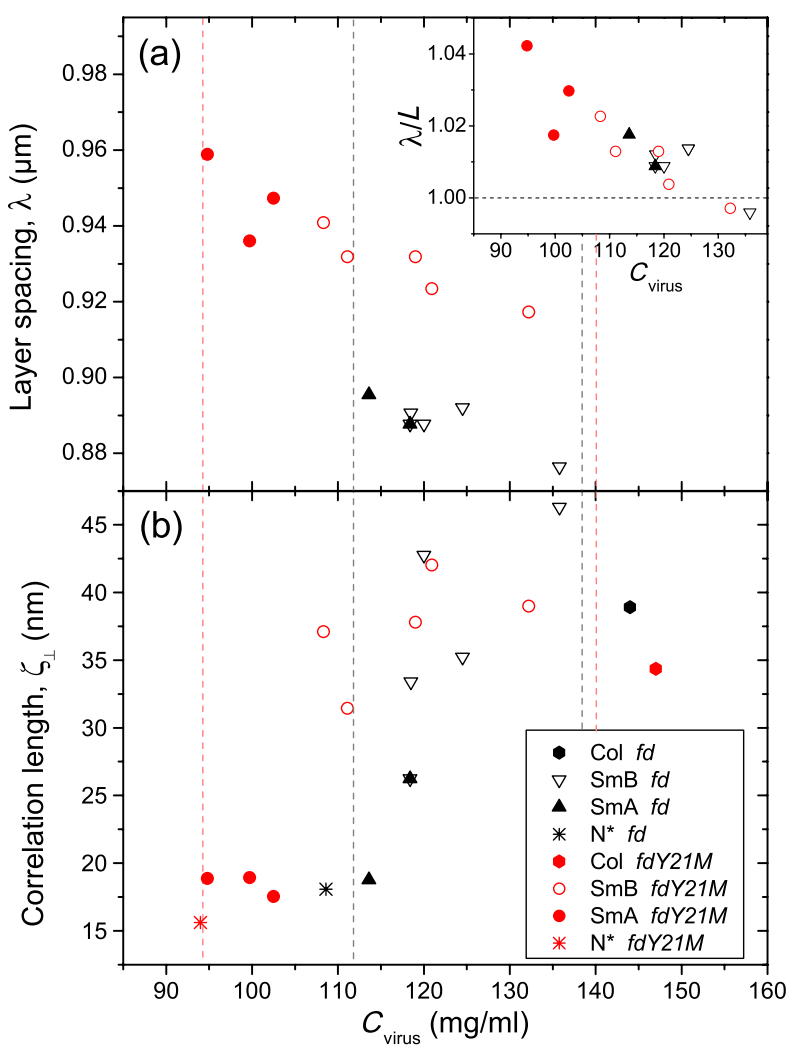

FIG. 6. (a) Smectic-layer spacing $\lambda$ obtained by fast Fourier transform of the optical microscopy images [Fig. 5(b)]. Inset: Smectic-layer spacing normalized by the respective viral contour length $L$. (b) Positional correlation length $\zeta_{\perp}=2 / F W H M$ within the smectic layer measured by SAXS on the (100) Bragg reflections [Fig. 5(c)] in the same samples as in (a). Black and red symbols refer to semiflexible $f d$ and stiff $f d Y 21 M$ viral rods, respectively. The dashed lines indicate the smectic range for $f d$ (gray) and $f d Y 21 M$ (red) suspensions $\left(I_{s}=20 \mathrm{mM}\right)$.

geometrical frustration between the hexagonal ordering and the helical twist between chiral viral particles [31].

The smectic-B phase also differs from a crystalline phase (Cr) for which crossed Bragg reflections are expected, indicating a strong coupling between the positional order within and between the layers. Moreover, it should be stressed that a crystalline phase has been observed beyond the columnar state after a slow drying of virus suspensions [31], then defining the following phase sequence by increasing virus concentration:

$$
\mathrm{I}-\mathrm{N}^{*}-\mathrm{SmA}-\mathrm{SmB}-\mathrm{Col}-\mathrm{Cr} .
$$

The occurrence of a columnar mesophase in between the smectic-B and the crystalline phases may be explained both by rod flexibility [27] acting as rod polydispersity and by a generic charge-induced stabilization of the columnar state, as explicitly demonstrated in different theoretical works $[29,44,45]$.
More quantitatively, the layer spacing $\lambda$ has been measured in the whole smectic range [Fig. 6(a)] and correlated with SAXS data to determine the nature of the smectic order (SmA or SmB). Because of the confinement within the layers induced by the high-rod density, the viruses are stretched and exhibit an effective end-to-end length distance very similar to their respective contour lengths $L$ [65]. Therefore, the layer spacing $\lambda$ normalized by the respective virus contour length $L$ is remarkably rescaled into a master curve for both filamentous particles of different stiffness $P$ [inset of Fig. 6(a)]. Moreover, $\lambda / L$ decreases by increasing rod concentration and reaches the value of $\lambda / L \simeq 1$ at the Sm-Col phase transition, which is shown to occur at the same concentration no matter what the rod flexibility is (Fig. 6). This is in contrast to the N-Sm transition for which the entropic penalty associated with the rod rigidity favors the smectic organization at lower rod densities $[20,27,36,64]$, leading to a broader smectic range for stiffer viruses (Fig. 6). Nevertheless, the fraction of SmA phase is similar for both viral strains, and it represents around $1 / 4$ of the whole smectic range. It is worth mentioning that the dynamical studies previously reported on the smectic state $[36,41,66]$ were all performed in the SmA phase.

\section{CONCLUSIONS}

In conclusion, our study shows that the electrostatic interaction of highly concentrated charged rods can be accounted for with the simple concept of an effective hardcore diameter $D_{\text {eff }}$. This heuristic model confirms that the $\mathrm{N}-\mathrm{Sm}$ transition location is universal in the long-rod limit and that it is only slightly sensitive to the softness of the interparticle potential. Though there is already extensive evidence from theory and computer simulations that phase transitions can be driven by entropy rather than enthalpy, our results are the first experimental demonstration that packing effects are dominant even for the increasingly complex ordered phases, which are the smectic phases. Furthermore, by varying rod stiffness thanks to viral mutants, we provide a reliable quantitative sketch of the competitive stability of the nematic, smectic-A, newly evidenced smectic-B, and columnar mesophases, which should stimulate further theoretical investigations and computer simulations where both rod flexibility and electrostatic interactions are included.

\section{ACKNOWLEDGMENTS}

This research is supported by CNRS. We thank Carlos Drummond and Seth Fraden for stimulating discussion.

[1] A. Einstein, On the Movement of Small Particles Suspended in Stationary Liquids Required by the Molecular Kinetic Theory of Heat, Ann. Phys. (Berlin) 322, 549 (1905). 
[2] P. N. Pusey and W. van Megen, Phase Behaviour of Concentrated Suspensions of Nearly Hard Colloidal Spheres, Nature (London) 320, 340 (1986).

[3] V. J. Anderson and H. N. W. Lekkerkerker, Insights into Phase Transition Kinetics from Colloid Science, Nature (London) 416, 811 (2002).

[4] A. Yethiraj and A. van Blaaderen, A Colloidal Model System with an Interaction Tunable from Hard Sphere to Soft and Dipolar, Nature (London) 421, 513 (2003).

[5] Z. Dogic and S. Fraden, in Soft Matter, edited by G. Gompper and M. Schick (Wiley-VCH, Weinheim, 2006), Vol. 2, pp. 1-86.

[6] F. C. Bawden, N. W. Pirie, J. D. Bernal, and I. Fankuchen, Liquid Crystalline Substances from Virus-Infected Plants, Nature (London) 138, 1051 (1936).

[7] H. Maeda and Y. Maeda, Liquid Crystal Formation in Suspensions of Hard Rodlike Colloidal Particles: Direct Observation of Particle Arrangement and Self-Ordering Behavior, Phys. Rev. Lett. 90, 018303 (2003).

[8] P. Davidson and J. C. P. Gabriel, Mineral Liquid Crystals, Curr. Opin. Colloid Interface Sci. 9, 377 (2005).

[9] G. J. Vroege, D. M. E. Thies-Weesie, A. V. Petukhov, B. J. Lemaire, and P. Davidson, Smectic Liquid-Crystalline Order in Suspensions of Highly Polydisperse Goethite Nanorods, Adv. Mater. 18, 2565 (2006).

[10] D. Kleshchanok, P. Holmqvist, J.-M. Meijer, and H. N. W. Lekkerkerker, Lyotropic Smectic B Phase Formed in Suspensions of Charged Colloidal Platelets, J. Am. Chem. Soc. 134, 5985 (2012).

[11] A. Kuijk, D. V. Byelov, A. V. Petukhov, A. van Blaaderen, and A. Imhof, Phase Behavior of Colloidal Silica Rods, Faraday Discuss. 159, 181 (2012).

[12] M. Kleman and O. D. Lavrentovich, Soft Matter Physics, An Introduction (Springer, New York, 2003).

[13] E. Barry and Z. Dogic, Entropy Driven Self-Assembly of Nonamphiphilic Colloidal Membranes, Proc. Natl. Acad. Sci. U.S.A. 107, 10348 (2010).

[14] Y. Yang, E. Barry, Z. Dogic, and M. Hagan, Self-Assembly of 2D Membranes from Mixtures of Hard Rods and Depleting Polymers, Soft Matter 8, 707 (2012).

[15] T. Gibaud, E. Barry, M. Zakhary, M. Henglin, A. Ward, Y. Yang, C. Berciu, R. Oldenbourg, M. Hagan, D. Nicastro, R. Meyer, and Z. Dogic, Reconfigurable Self-Assembly through Chiral Control of Interfacial Tension, Nature (London) 481, 348 (2012).

[16] L. Onsager, The Effects of Shape on the Interaction of Colloidal Particles, Ann. N.Y. Acad. Sci. 51, 627 (1949).

[17] D. Frenkel, H. N. W. Lekkerkerker, and A. Stroobants, Thermodynamic Stability of a Smectic Phase in a System of Hard Rods, Nature (London) 332, 822 (1988).

[18] J.-L. Barrat and J.-P. Hansen, Basic Concepts for Simple and Complex Liquids (Cambridge University Press, Cambridge, England, 2003).

[19] A. Poniewierski, Nematic to Smectic-A Transition in the Asymptotic Limit of Very Long Hard Spherocylinders, Phys. Rev. A 45, 5605 (1992).

[20] A. V. Tkachenko, Nematic-Smectic Transition of Semiflexible Chains, Phys. Rev. Lett. 77, 4218 (1996).

[21] P. Bolhuis and D. Frenkel, Tracing the Phase Boundaries of Hard Spherocylinders, J. Chem. Phys. 106, 666 (1997).
[22] J. D. Parsons, Nematic Ordering in a System of Rods, Phys. Rev. A 19, 1225 (1979).

[23] S. D. Lee, A Numerical Investigation of Nematic Ordering Based on a Simple Hard-Rod Model, J. Chem. Phys. 87, 4972 (1987).

[24] B. Mulder, Density-Functional Approach to Smectic Order in an Aligned Hard-Rod Fluid, Phys. Rev. A 35, 3095 (1987).

[25] A. M. Somoza and P. Tarazona, Nematic and Smectic Liquid Crystals of Hard Spherocylinders, Phys. Rev. A 41, 965 (1990).

[26] A. M. Bohle, R. Holyst, and T. Vilgis, Polydispersity and Ordered Phases in Solutions of Rodlike Macromolecules, Phys. Rev. Lett. 76, 1396 (1996).

[27] P. van der Schoot, The Nematic-Smectic Transition in Suspensions of Slightly Flexible Hard Rods, J. Phys. II (France) 6, 1557 (1996).

[28] J. M. Polson and D. Frenkel, First-Order Nematic-Smectic Phase Transition for Hard Spherocylinders in the Limit of Infinite Aspect Ratio, Phys. Rev. E 56, R6260 (1997).

[29] H. H. Wensink, Columnar versus Smectic Order in Systems of Charged Colloidal Rods, J. Chem. Phys. 126, 194901 (2007).

[30] Z. Dogic and S. Fraden, Smectic Phase in a Colloidal Suspension of Semiflexible Virus Particles, Phys. Rev. Lett. 78, 2417 (1997).

[31] E. Grelet, Hexagonal Order in Crystalline and Columnar Phases of Hard Rods, Phys. Rev. Lett. 100, 168301 (2008).

[32] O. J. Dammone, I. Zacharoudiou, R. P. A. Dullens, J. M. Yeomans, M. P. Lettinga, and D. G. A. L. Aarts, Confinement Induced Splay-to-Bend Transition of Colloidal Rods, Phys. Rev. Lett. 109, 108303 (2012).

[33] Y. A. Wang, X. Yu, S. Overman, M. Tsuboi, G. J. Thomas Jr., and E. H. Egelman, The Structure of a Filamentous Bacteriophage, J. Mol. Biol. 361, 209 (2006).

[34] E. Barry, D. Beller, and Z. Dogic, A Model Liquid Crystalline System Based on Rodlike Viruses with Variable Chirality and Persistence Length, Soft Matter 5, 2563 (2009).

[35] W. M. Tan, R. Jelinek, S. J. Opella, P. Malik, T. D. Terry, and R. N. Perham, Effects of Temperature and Y21 M Mutation on Conformational Heterogeneity of the Major Coat Protein (pVIII) of Filamentous Bacteriophage fd, J. Mol. Biol. 286, 787 (1999).

[36] E. Pouget, E. Grelet, and M. P. Lettinga, Dynamics in the Smectic Phase of Stiff Viral Rods, Phys. Rev. E 84, 041704 (2011).

[37] Z. Zhang and E. Grelet, Tuning Chirality in the Self-Assembly of Rod-like Viruses by Chemical Surface Modifications, Soft Matter 9, 1015 (2013).

[38] K. Zimmermann, H. Hagedorn, C. Chr. Heucks, M. Hinrichsen, and H. Ludwig, The Ionic Properties of the Filamentous Bacteriophages Pfl and fd, J. Biol. Chem. 261, 1653 (1986).

[39] J. X. Tang, P. A. Janmey, A. Lyubartsev, and L. Nordenskiöld, Metal Ion-Induced Lateral Aggregation of Filamentous Viruses fd and M13, Biophys. J. 83, 566 (2002).

[40] K. Kang, A. Wilk, A. Patkowski, and J. K. G. Dhont, Diffusion of Spheres in Isotropic and Nematic Networks of Rods: Electrostatic Interactions and Hydrodynamic Screening, J. Chem. Phys. 126, 214501 (2007).

[41] E. Grelet, M. P. Lettinga, M. Bier, R. van Roij, and P. van der Schoot, Dynamical and Structural Insights into the 
Smectic Phase of Rod-like Particles, J. Phys. Condens. Matter 20, 494213 (2008).

[42] K. R. Purdy, and S. Fraden, Influence of Charge and Flexibility on Smectic Phase Formation in Filamentous Virus Suspensions, Phys. Rev. E 76, 011705 (2007).

[43] G. Nägele, in The Physics of Colloidal Soft Matter, Lecture Notes Vol. 14 (Polish Academy of Sciences Publication, Warsaw, 2004).

[44] H. Graf and H. Löwen, Phase Diagram of Tobacco Mosaic Virus Solutions, Phys. Rev. E 59, 1932 (1999).

[45] E. M. Kramer and J. Herzfeld, Avoidance Model for Soft Particles. II. Positional Ordering of Charged Rods, Phys. Rev. E 61, 6872 (2000).

[46] M. N. Tamashiro and H. Schiessel, Where the Linearized Poisson-Boltzmann Cell Model Fails: Spurious Phase Separation in Charged Colloidal Suspensions, J. Chem. Phys. 119, 1855 (2003).

[47] G. S. Manning, Limiting Laws and Counterion Condensation in Polyelectrolyte Solutions, J. Chem. Phys. 51, 924 (1969).

[48] G. S. Manning, Ionic Polarizability of Interacting Charged Rods, Europhys. Lett. 86, 36001 (2009).

[49] G. S. Manning, Counterion Condensation on Charged Spheres, Cylinders, and Planes, J. Phys. Chem. B 111, 8554 (2007).

[50] M. Deserno, C. Holm, and S. May, Fraction of Condensed Counterions around a Charged Rod: Comparison of Poisson-Boltzmann Theory and Computer Simulations, Macromolecules 33, 199 (2000).

[51] E. Trizac, L. Bocquet, and M. Aubouy, Simple Approach for Charge Renormalization in Highly Charged Macroions, Phys. Rev. Lett. 89, 248301 (2002).

[52] L. Bocquet, E. Trizac, and M. Aubouy, Effective Charge Saturation in Colloidal Suspensions, J. Chem. Phys. 117, 8138 (2002).

[53] E. Trizac, M. Aubouy, and L. Bocquet, Analytical Estimation of Effective Charges at Saturation in PoissonBoltzmann Cell Models, J. Phys. Condens. Matter 15, S291 (2003).

[54] E. Eggen, M. Dijkstra, and R. van Roij, Effective Shape and Phase Behavior of Short Charged Rods, Phys. Rev. E 79, 041401 (2009).
[55] A. Stroobants, H. N. W. Lekkerkerker, and Th. Odjik, Effect of Electrostatic Interaction on the Liquid Crystal Phase Transition in Solutions of Rodlike Polyelectrolytes, Macromolecules 19, 2232 (1986).

[56] G. J. Vroege and H. N. W. Lekkerkerker, Phase Transitions in Lyotropic Colloidal and Polymer Liquid Crystals, Rep. Prog. Phys. 55, 1241 (1992).

[57] K. R. Purdy and S. Fraden, Isotropic-Cholesteric Phase Transition of Filamentous Virus Suspensions as a Function of Rod Length and Charge, Phys. Rev. E 70, 061703 (2004).

[58] J. Tang and S. Fraden, Isotropic-Cholesteric Phase Transition in Colloidal Suspensions of Bacteriophage fd, Liq. Cryst. 19, 459 (1995).

[59] K. R. Purdy, Z. Dogic, S. Fraden, A. Rühm, L. Lurio, and S. G. J. Mochrie, Measuring the Nematic Order of Suspensions of Colloidal fd Virus by X-ray Diffraction and Optical Birefringence, Phys. Rev. E 67, 031708 (2003).

[60] Z. Y. Chen, Nematic Ordering in Semiflexible Polymer Chains, Macromolecules 26, 3419 (1993).

[61] B. Martinez-Haya and A. Cuetos, Stability of Nematic and Smectic Phases in Rod-Like Mesogens with OrientationDependent Attractive Interactions, J. Phys. Chem. B 111, 8150 (2007).

[62] A. Yethiraj, Tunable Colloids: Control of Colloidal Phase Transitions with Tunable Interactions, Soft Matter 3, 1099 (2007).

[63] G. Cinacchi and L. de Gaetani, Phase Behavior of Wormlike Rods, Phys. Rev. E 77, 051705 (2008).

[64] R. C. Hidalgo, D. E. Sullivan, and J. Z. Y. Chen, Smectic Ordering of Homogeneous Semiflexible Polymers, Phys. Rev. E 71, 041804 (2005).

[65] Z. Dogic, J. Zhang, A. W. C. Lau, H. Aranda-Espinoza, P. Dalhaimer, D. E. Discher, P. A. Janmey, R. D. Kamien, T. C. Lubensky, and A. G. Yodh, Elongation and Fluctuations of Semiflexible Polymers in a Nematic Solvent, Phys. Rev. Lett. 92, 125503 (2004).

[66] M. P. Lettinga and E. Grelet, Self-Diffusion of Rodlike Viruses through Smectic Layers, Phys. Rev. Lett. 99, 197802 (2007). 